\title{
Protocol for The International Cohort on Lifestyle Determinants of Health Study: A Longitudinal Investigation of Complementary and Integrative Health Utilization in Postsecondary Education Students
}

\author{
Ryan Bradley, ND, MPH, ${ }^{1-3}$ Courtney K. Pickworth, ND, MS, Ryan S. Wexler, BS, \\ Adam Sadowski, ND, MS, Lita Buttolph, PhD, DSOM, ${ }^{1}$ Hadil Sarrar, BA, Jillian Moehle, BS, \\ M. Tediana Torrens, $\mathrm{BA}^{1}$ Joanna Harnett, $\mathrm{PhD}_{1}^{3,4}$ Erica Mclntyre, $\mathrm{PhD}^{4}$ Janet Schloss, $\mathrm{PhD}^{4,5}$ \\ Amie Steel, ND, $\mathrm{PhD}, \mathrm{MPH}^{4}$ and Jon Adams, $\mathrm{PhD}^{4}$
}

\begin{abstract}
Objectives: The specific aims are: 1) To characterize the health, wellness, and lifestyle of graduate and undergraduate students, and how these characteristics change over time; 2) To evaluate associations between lifestyle factors and gut microbiota populations and diversity; and 3) To evaluate associations between stress and stress management practices with sleep habits, quality of life, and overall health.

Design: The International Cohort on Lifestyle Determinants of Health (INCLD Health) longitudinal cohort study is designed to assess health behaviors and lifestyle practices amongst adults studying complementary and integrative health $(\mathrm{CIH})$ and higher-education students more generally after at least one to six years of exposure to $\mathrm{CIH}$ education. INCLD Health will adhere to the Strengthening the Reporting of Observational studies in Epidemiology (STROBE) guidelines.

Settings/Location: Colleges and universities with a $\mathrm{CIH}$ focus or interest with the flagship site being the National University of Natural Medicine.

Participants: Adults currently enrolled in a college or university with a CIH focus or interest.

Outcome Measures: Study visits will be conducted at baseline, 6 months, then every 12 months until the end of each participants' degree program. Measures include anthropometrics; serum and salivary biomarkers of cardiovascular risk, reproductive hormones, and cortisol; nutritional intake measured by a digital food frequency questionnaire; sequencing of fecal microbiota; plus validated questionnaires investigating mood, perceived stress, stress management practices, physical activity, sleep, and wellness.

Conclusions: The INCLD Health Study, approved by the NUNM IRB in late 2018, will enroll a unique cohort of adults to characterize the use of $\mathrm{CIH}$ practices in relation to short- and long-term health. Our study design provides a breadth of information that could be implemented at multiple sites internationally allowing for comparisons across diverse student cohorts with relatively low cost and personnel.
\end{abstract}

\footnotetext{
${ }^{1}$ Helfgott Research Institute, National University of Natural Medicine, Portland, OR, USA.

${ }^{2}$ Family Medicine and Public Health, University of California, San Diego, San Diego, CA, USA.

${ }^{3}$ School of Pharmacy, Faculty of Medicine and Health, The University of Sydney, Sydney, Australia.

${ }^{4}$ Australian Research Centre of Complementary and Integrative Medicine, Faculty of Health, University of Technology Sydney, Ultimo, Australia.

${ }^{5}$ National Centre for Naturopathic Medicine, Southern Cross University, Lismore, Australia.
} 
Keywords: cohort studies, complementary and integrative health, nutritional status, self care, health behavior, lifestyle, gastrointestinal microbiome, cardiovascular disease

\section{Introduction}

\section{Background and rationale}

$\mathbf{T}$ He World Health Organization estimates that $60 \%$ of factors related to health and quality of life are correlated with lifestyle. ${ }^{1}$ The term "healthy lifestyle" encompasses a number of modifiable lifestyle choices including, but not limited to, dietary factors, physical activity, minimizing substance use, and improving sleep habits. Research surrounding healthy lifestyle choices suggests that certain modifiable lifestyle behaviors have far-reaching effects on future health, physical function, and all-cause mortality. ${ }^{2-4}$

The Centers for Disease Control (CDC) conducts regular population-based studies, including the National Health Interview Survey (NHIS) and National Health and Nutrition Examination Survey (NHANES) to assess health exposures and outcomes in a large cohort sample of the population of the United States. ${ }^{5,6}$ Data from these surveys have been instrumental in the development of guidelines surrounding diet, physical activity, and other contributors to health. ${ }^{7}$ In addition to NHIS and NHANES, other longitudinal studies designed to assess exposures and health outcomes have been conducted in large cohorts of health professionals. Analysis of data gathered in the Nurses' Health Study (NHS) and Health Professionals Follow-up Study (HPFS) has led to numerous studies investigating associations between diet, lifestyle, and health habits with chronic disease risk, cancer, and reproductive health. ${ }^{8-11}$ Currently, the CDC dedicates a large section of its website to information on "healthy living" with resources for the general public and health care practitioners with the goal of promoting healthy behaviors in the general population; despite the availability of this information, only $20 \%$ of Americans meet the physical activity recommendations and less than $10 \%$ meet nutritional guidelines. ${ }^{12,13}$

Health behaviors are often a matter of personal preference or are dictated by the available resources; therefore, assessing exposures and outcomes in defined subgroups could contribute to the evidence base that informs lifestyle and health-related guidelines in specific populations. Depending on exposure, different communities may engage in health and lifestyle behaviors that do not align with clinical and public health guidelines. For example, students of complementary and integrative health $(\mathrm{CIH})$ programs may participate in and/or subscribe to a unique set of lifestyle behaviors in response to their educational content (e.g., nutrition, herbal medicine, and mind-body practices) that are not routinely available in academic programs for other health disciplines. ${ }^{14,15}$ These behaviors have not been well-documented in the literature and represent a valuable source of information on health practices that are often outside of the "mainstream" or may be contrary to conventional recommendations.

The International Cohort on Lifestyle Determinants of Health (INCLD Health) study represents an opportunity to gather such data in a manner similar to NHIS and HPFS. Prospective longitudinal cohort trials offer several benefits, including the ability to identify specific exposures/or behaviors and follow participants over time. ${ }^{16}$ This longitudinal cohort study will provide investigators with a range of data regarding determinants of health associated with lifestyle behaviors and CIH utilization among students - a population that is currently unstudied.
Therefore, the authors designed a protocol for a prospective longitudinal cohort study that will collect information about such students. Data captured in this study will include dietary practices, mental health, and physical activity practices using validated measures; cardiometabolic risk using salivary and serum samples; gut microbiome diversity using fecal samples; and a comprehensive medical history review, as well as information about $\mathrm{CIH}$ practices and beliefs using novel measures. In this study, the authors present the methodological design and study protocol for the INCLD Health study.

\section{Aims and objectives}

The primary aim of this study is to characterize the impact of CIH utilization and practices on physical and mental health and perceived wellness on higher education students over time. The second aim is to determine the influence of nutritional, mind-body practices and stress on the composition and diversity of the gut microbiome. The third aim is to evaluate student's perceived stress, sleep quality and quantity, and/or physical functioning, as indicators of health-related quality of life. In addition, the INCLD Health Study is similar to other longitudinal cohort studies in that it will support many ancillary investigations, including comparisons to other cohorts.

\section{Hypotheses}

The authors hypothesize that adults exposed to $\mathrm{CIH}$ education will engage in more $\mathrm{CIH}$-related practices compared to other populations, including student cohorts of similar age and non-CIH health degrees. Furthermore, $\mathrm{CIH}$ students engaging in more $\mathrm{CIH}$ practices at baseline and over time will correlate with lower perceived stress levels, a more diverse gut microbiome, better sleep quality and mood, higher healthrelated quality of life and personal wellness, and a lower cardiometabolic risk compared to other cohorts of a similar demographic where the exposure to $\mathrm{CIH}$ practices is less.

\section{Materials and Methods}

The flagship site for this study is the National University of Natural Medicine (NUNM) in Portland, Oregon. This study has been approved by the NUNM Institutional Review Board (approval no.: RB091218). All further publications upon sufficient data collection, as they relate to INCLD Health, will strictly adhere to the strengthening the reporting of observational studies in epidemiology (STROBE) guidelines. ${ }^{17}$

\section{Target population}

The target population for this study is adults (18 years old and above) currently enrolled in $\mathrm{CIH}$ education programs. By utilizing adults exposed to $\mathrm{CIH}$-focused modalities and philosophies, the authors will capture a unique population of students with high $\mathrm{CIH}$ exposure. Participant inclusion and exclusion criteria are outlined in Table 1.

\section{Recruitment}

Participants will be initially recruited from the student population at NUNM and will be extended to student health 
TABle 1. InClusion AND ExClusion CRiteria

Inclusion criteria

Exclusion criteria

Currently enrolled in complementary medicine education program Not meeting inclusion criteria

Willingness to complete baseline, 6-month, and annual follow-up Students in the final year of their program with the

study visits, including a minimum of data collection

of demographic information, vital signs, and online surveys

Able to provide informed consent

Able to read, write, and understand English

and wellness programs affiliated with accredited schools across the globe. Recruitment will utilize primarily convenience sampling of the student population through acceptable methods on campus: bulletin board postings, e-mails, social media, and the school website. In addition, a website for INCLD Health will be designed using Microsoft SharePoint for prospective and current participants to access information about the study. Participants will be enrolled in the study on a rolling basis and comprise a single cohort moving forward. Students interested in participating will arrange a time for online screening with a study coordinator and, if eligible and willing to consent, are then signed up for a baseline study visit. Incentives for participation in the study will include reports on their laboratory and microbiota results, a detailed nutritional analysis of their dietary pattern, and a free reusable INCLD Health-logoed mug.

\section{Procedures}

All study visits for the flagship cohort will be conducted at the NUNM Helfgott Research Institute or online through Zoom. All study visits for other institutions will conduct their own screening at their campus or virtually if necessary. Study procedures for each visit are outlined in the study timeline presented in Table 2.

Study visit one (baseline). Participants who meet the eligibility criteria during their e-mail screening will be scheduled for a baseline visit, which will take $90 \mathrm{~min}$ to complete. During this visit, participants will review the informed consent with study personnel and sign the document if they would like to participate, with an understanding that surveys may contain sensitive information that the participant may not want to disclose. Participants will be informed that they have the option to skip surveys if they do not wish to disclose information and can still maintain participation throughout the study. Procedures at the visit will include anthropometric measurements (self-reported in the case of online visits); questionnaires on REDCap ${ }^{\circledR}$ software regarding demographic information, lifestyle choices, and wellness;

\section{Table 2. Cardiometabolic Markers} FROM ZRT LABORATORY

Blood Thyroid stimulating hormone, insulin, glycosylated hemoglobin, vitamin $\mathrm{D}$, high sensitivity

C-reactive protein, triglycerides, total cholesterol, high density lipoprotein, low density lipoprotein, and very low density lipoprotein.

Saliva Estradiol, progesterone, testosterone, dehydroepiandrosterone, cortisol $(\times 4)$ a validated online food frequency questionnaire (i.e., Vioscreen $^{\text {TM }}$ by VioCare, Princeton, NJ, USA); and review instructions for optional at-home collections of blood, saliva, and stool specimens for the ZRT Laboratory "Weight Management Profile with Cardiovascular Markers" Kit and the American Gut Project Kit. Participants who are unable to complete the questionnaire during their visit will be provided remote access to complete their REDCap surveys within 1 week of the baseline visit. Participants who do not complete their surveys within 1 week of the baseline visit will be reminded to do so through their preferred e-mail.

Participants will be asked to return self-collected specimens to the Helfgott Research Institute within 1 month, with specimens accepted up to 3 months from the baseline visit date. Blood and salivary specimens are shipped quarterly to ZRT laboratories, and stool specimens are shipped annually to be sequenced.

Study visit two (6-month follow-up). The first follow-up visit will take place 6 months from each participant's baseline visit; this study visit must be complete within 1 month of the estimated date and will take $60 \mathrm{~min}$ to complete. This visit will include all previous procedures (anthropometric measurements, REDCap surveys, VioScreen questionnaires, and ZRT at-home salivary and blood sample collection) except for stool specimen collection. Similarly, participants who do not complete their surveys within 1 week of the 6th month visit will be reminded to do so through their preferred e-mail.

Study visits three to eight (annual follow-ups). The participants will be scheduled for their first annual follow-up visit 1 year from their baseline visit and undergo all previous procedures (anthropometric measurements, REDCap surveys, VioScreen questionnaires, and ZRT at-home salivary and blood sample collection) with the addition of the American Gut Project stool specimen collection. Visits will then be conducted on an annual basis for the remainder of their enrollment. Participants will be considered discharged from the study once they have completed a follow-up within their last year of matriculation; however, they will have the opportunity to continue providing annual follow-up data if interested.

\section{Outcome measures}

Study personnel will administer several validated and supplemental questionnaires at each visit. Surveys will be self-reported assessments of overall health, quality of life, mental health, and lifestyle habits. Supplemental measures will be created by the study team to assess lifestyle factors 
commonly emphasized within the CIH community (e.g., utilization of organic produce, medicinal herbs in cooking, health effects of fermented foods, choice of cooking oils, and so on).

International Physical Activity Questionnaire. The International Physical Activity Questionnaire (IPAQ) has been shown to have high repeatability, with $75 \%$ of the Spearman correlation coefficients observed in a 12-country validation study to be above 0.65 and ranging from 0.88 to $0.32 .{ }^{18}$ The IPAQ also demonstrated high criterion validity ranging from 0.14 to 0.53 , with a median of about $0.30 .^{19}$ Reliability and validity have also been assessed in other languages and populations both healthy and unhealthy. ${ }^{20-22}$ The IPAQ is a 12-question validated tool designed to capture information about a range of physical activity frequency, intensity, time, and type. Additional questions regarding physical activity and exercise will be assessed using a supplemental exercise survey developed by study personnel. To score the IPAQ, questions are summed within each physical activity domain to estimate the total time spent in occupational, transport, household, and leisure-related physical activity, as well as total time spent sitting. Weekly physical activity participation is then calculated by multiplying days/week of participation by the amount of time/day and finally by a MET (metabolic equivalent) value assigned to each activity intensity.

Major Depression Inventory. To further assess for depressive symptoms, the Major Depression Inventory (MDI) will be administered at each study visit. The MDI is a 10 -item survey assessing depression symptoms that are validated to correspond with major depressive disorder according to the Diagnostic \& Statistical Manual of Mental Disorders (DSM) IV with high sensitivity (90\%) and specificity $(80 \%) .^{23}$ The MDI has been found to be reliable in multiple populations internationally, including patients with neurologic impairment and other conditions. ${ }^{24,25}$ Question responses are on a five-point Likert scale where zero indicates "at no time" and five indicates "all the time." In the event that participants indicate high scores on questions pertaining directly to depressed mood and suicidal ideation, they will be provided with resources to seek help in crisis and to establish care with a provider.

Patient Reported Outcome Measures Information System 29. The Patient Reported Outcome Measures Information System 29 (PROMIS-29) will assess physical function, anxiety, depression, fatigue, sleep disturbance, ability to participate in social roles and activities, pain interference, and pain intensity. Overall, the PROMIS-29 short forms demonstrated high reliability compared with the long form PROMIS questionnaires. ${ }^{26}$ The PROMIS-29 depression and anxiety subscales have been shown to have high inter-rater reliability (Cronbach's $\alpha=0.93$ and 0.89 ) compared to other validated metrics such as the Patient Health Questionnaire 9 (PHQ-9) and General Anxiety Disorder 7 (GAD-7), which are validated self-reported questionnaires frequently used in primary care settings for the classification of depression and anxiety symptoms, respectively. ${ }^{27}$ The physical and mental health scores of the PROMIS-29 have also been shown to be reliable in individuals with multiple chronic conditions when evaluated against the Veterans RAND 36-Item Health
Survey (Pearson's correlation coefficients $=0.85$ and 0.70). ${ }^{28}$ The PROMIS-29 consists of 29 questions. Seven domains are queried with four questions per domain, each reported on a five-point Likert scale. One additional question asks the user to rate their pain intensity on a scale from 0 to 10 . In general, the questions ask for participants to self-assess their function anchored to the last 7 days; however, the "physical function" and "social interaction" subscales are not anchored to a specific time frame. A raw score is calculated from each of the domains and then translated into T-scores. The Pain Intensity item is reported as a raw score. PROMIS has been shown to be a flexible, efficient, and precise tool for measuring common patient-reported outcomes.

PROMIS Sexual Function and Satisfaction v2.0 Brief Profile (female and male). The PROMIS SexFS Brief Profile measures sexual function and satisfaction for males and females across a range of categories such as sexual enjoyment, discomfort, lubrication, desire, arousal, and so on over the past 30 days. ${ }^{29}$ Responses are recorded on a fiveto six-point Likert scale, depending on the question, with 15 questions for females and 11 for males.

Perceived Stress Scale. The 10-item Perceived Stress Scale (PSS-10) is a validated tool designed to capture information regarding stress and stress management. It has been shown to be the most reliable perceived stress scale among the 4-, 10-, and 14-item variations with test-retest reliability consistently $>0.70$ using intraclass correlation. ${ }^{30}$ Participants respond to a series of 10 questions with 4 answer choices ranging from never to very often.

\section{Third-party samples}

Objective assessments of physiology (e.g., cardiovascular risk markers) will provide a significantly more complete and holistic view of participants' health and wellness. Additional data will be collected using third-party partners, including VioScreen, ZRT Laboratory, and the American Gut Project.

American Gut Project Microbiome analysis. The American Gut Project is an open-access, citizen-driven data repository based at the University of California, San Diego Center for Microbiome Innovation that provides intestinal microbiome analysis. Results will be uploaded to a secure online portal from which investigators will access them for each participant. Participants will receive information about the composition of bacterial populations in their stool samples from a list of commonly found microbes, as well as data on how their microbiome diversity compares to those of others in similar demographics. ${ }^{31}$

VioScreen dietary analysis. The VioScreen has been shown to be reliable in healthy adults ${ }^{25}$ and will be used to collect data about dietary patterns and nutritional choices. This questionnaire will be completed online using VioScreen's patient portal during the study visits. The questionnaire will collect 90 days' worth of dietary and nutritional data from participants. VioScreen will also provide nutrient analysis based on information from the Nutrient Coordinating Center. Results and analysis will be obtained directly from VioScreen. ${ }^{32,33}$ 
ZRT Laboratory weight management panel with cardiovascular add-ons. ZRT Laboratory (Beaverton, OR, USA) will provide "Weight Management Profile with Cardiovascular Markers" sample collection kits for all participants. Samples include one fasting blood spot, as well as four salivary cortisol samples collected throughout the day (waking, lunchtime, dinnertime, and evening). Samples from these kits will be analyzed by ZRT for many biomarkers of cardiometabolic, endocrine, and reproductive health (Table 3).

\section{Supplemental measures}

See Supplementary Appendix SA1 for copies of each measure.

Health History Questionnaire. The Health History Questionnaire (HHQ) assesses participants for 89 past or ongoing common acute and chronic diseases, including conditions within the following categories: cardiovascular, respiratory, dermatologic, gastrointestinal, neuromuscular, endocrine, genitourinary, cancer, and psychiatric, as well as providing space for participants to list other conditions not specified above. This measure was designed based on several clinic intake forms used by primary care clinics in the Portland, Oregon area, including the NUNM Health Clinic.

List of medications and supplements. Before the visit, participants will be asked to bring any of their current medications and supplements. The following information will be collected for each medication and supplement that the participant is currently taking or has taken in the past month: medication name, manufacturer name (supplements only), dose per unit (capsule, tablet, drop, etc.), dose unit (mg, mcg, g, etc.), number of days in the month taken, start date (mm/yyyy), front and back photo of the product label, and reason for use. Participants will be able to select from the following "Reason for Use" categories: stress, sleep, digestive, hormonal, cognition, musculoskeletal, antiinflammatory, immune, mood, respiratory, cardiac, skin, energy, weight management, general health, and specify other. The inclusion of participant supplement information in this detail is a novel aspect of this study that will allow for appropriate statistical control tactics, as well as future investigations of how supplements broadly and specifically impact health.

Multidimensional Index of Wellness. Self-reported wellness will be assessed while piloting a supplemental survey tool adapted from the Personal Wellbeing Index (PWI)Adult version (5th edition) by the International Wellbeing Group. $^{34}$ The authors adapted this measure to ask participants to rate their current level of wellness in 10 different subdomains: physical, emotional, mental, spiritual, sexual, personal/relation with self, familial/relation with family, social/relation with community, financial, and overall. Participants will rate these wellness dimensions on a scale of $1-10$, where 10 represents "very high," and 1 represents "very low."

Table 3. Study Visit Schedule

\begin{tabular}{|c|c|c|c|c|c|}
\hline & $\begin{array}{l}\text { E-mail screening } \\
\text { and scheduling }\end{array}$ & $\begin{array}{l}\text { Study visit } 1 \\
\text { (baseline) }\end{array}$ & $\begin{array}{l}\text { Study visit } 2 \\
\text { (6 months) }\end{array}$ & $\begin{array}{l}\text { Study visit } 3 \\
(12 \text { months })\end{array}$ & $\begin{array}{c}\text { Annual } \\
\text { study visits }\end{array}$ \\
\hline Eligibility criteria & $\mathrm{X}$ & & & & \\
\hline Informed consent & $\mathrm{X}$ & $\mathrm{X}$ & & & \\
\hline $\begin{array}{l}\text { American Gut Project stool sample collection } \\
\text { kit and instructions }\end{array}$ & & $X$ & & $\mathrm{X}$ & $\mathrm{X}$ \\
\hline Biometric measurements & & $\mathrm{X}$ & $\mathrm{X}$ & $\mathrm{X}$ & $\mathrm{X}$ \\
\hline Demographic information questionnaire & & $\mathrm{X}$ & $\mathrm{X}$ & $\mathrm{X}$ & $\mathrm{X}$ \\
\hline Health history questionnaire & & $\mathrm{X}$ & $\mathrm{X}$ & $\mathrm{X}$ & $\mathrm{X}$ \\
\hline International physical activity questionnaire & & $\mathrm{X}$ & $\mathrm{X}$ & $\mathrm{X}$ & $\mathrm{X}$ \\
\hline Major depression inventory & & $\mathrm{X}$ & $\mathrm{X}$ & $\mathrm{X}$ & $\mathrm{X}$ \\
\hline $\begin{array}{l}\text { Medication and supplement interviewer-assisted } \\
\text { questionnaire }\end{array}$ & & $\mathrm{X}$ & $\mathrm{X}$ & $\mathrm{X}$ & $X$ \\
\hline Multidimensional index of wellness & & $\mathrm{X}$ & $\mathrm{X}$ & $\mathrm{X}$ & $\mathrm{X}$ \\
\hline Long-term use of medications & & $\mathrm{X}$ & $\mathrm{X}$ & $\mathrm{X}$ & $\mathrm{X}$ \\
\hline $\begin{array}{l}\text { Patient reported outcome measures information } \\
\text { system sexual function profile }\end{array}$ & & $X$ & $\mathrm{X}$ & $\mathrm{X}$ & $\mathrm{X}$ \\
\hline $\begin{array}{l}\text { Patient reported outcome measures information } \\
\text { system-29 }\end{array}$ & & $\mathrm{X}$ & $\mathrm{X}$ & $\mathrm{X}$ & $\mathrm{X}$ \\
\hline Perceived stress scale & & $\mathrm{X}$ & $\mathrm{X}$ & $\mathrm{X}$ & $\mathrm{X}$ \\
\hline Reproductive status & & $\mathrm{X}$ & $\mathrm{X}$ & $\mathrm{X}$ & $\mathrm{X}$ \\
\hline Stress management and self-care survey & & $\mathrm{X}$ & $\mathrm{X}$ & $\mathrm{X}$ & $\mathrm{X}$ \\
\hline Substance use survey & & $\mathrm{X}$ & $\mathrm{X}$ & $\mathrm{X}$ & $\mathrm{X}$ \\
\hline Supplemental dietary intake survey & & $\mathrm{X}$ & $\mathrm{X}$ & $\mathrm{X}$ & $\mathrm{X}$ \\
\hline Supplemental physical activity survey & & $\mathrm{X}$ & $\mathrm{X}$ & $\mathrm{X}$ & $\mathrm{X}$ \\
\hline Symptom monitoring questionnaire & & $\mathrm{X}$ & $\mathrm{X}$ & $\mathrm{X}$ & $\mathrm{X}$ \\
\hline VioScreen ${ }^{\mathrm{TM}}$ questionnaire & & $\mathrm{X}$ & $\mathrm{X}$ & $\mathrm{X}$ & $\mathrm{X}$ \\
\hline $\begin{array}{l}\text { ZRT laboratory sample collection kit and } \\
\text { instructions }\end{array}$ & & $X$ & $X$ & $X$ & $X$ \\
\hline Estimated duration & $5 \mathrm{~min}$ & $90 \mathrm{~min}$ & $60 \mathrm{~min}$ & $60 \mathrm{~min}$ & $60 \mathrm{~min}$ \\
\hline
\end{tabular}


Stress management and self-care survey. This survey applies the question structure from the NHIS ${ }^{35}$ and extends to other types of self-care activities. This eight-question survey asks what kinds of self-care practices participants used in the past month and the frequency of those practices ranging from "never" to "more than 3 times per week." These include mindfulness meditation, prayer, asana yoga, pranayama yoga, t'ai chi or qigong, and other, which can be detailed by participants. As students possibly engage in those practices which they are learning about, the authors will provide the option for participants to report participation in many stress management and self-care habits that they may have been exposed to in their respective programs.

Substance use survey. Participants will be asked six questions to provide information regarding their current substance use habits, including frequency, "never" to " 2 or more times per day," and type of substances used: tobacco, e-cigarettes/vaping, cannabis, recreational drugs, and alcohol, as a correlate of mental health and negative coping mechanism(s).

Supplemental dietary intake survey. This survey applies the question structure from the NHANES ${ }^{36}$ and extends to other types of foods and eating behaviors not captured in the VioScreen food frequency questionnaires (FFQ). In this survey, participants will be asked 150 questions about food choices and frequency of those choices that will provide additional insight into their health food behaviors. These questions include participants' dietary preferences, (vegetarian, vegan, gluten-free, etc.) and consumption of various organic products, fermented foods, grains, antioxidant-rich foods and beverages, culinary herbs and spices, alcohol, cooking oils and fats, uncooked oils and fats, and international foods. Participants will also be asked to report what foods they avoid and how they prepare their meals (i.e., from scratch, prepackaged, or dining out).

Supplemental physical activity survey. This survey applies the question structure from the IPAQ and extends to other activities by asking participants to report their frequency of participation, level of intensity, and duration of participation in a broader range of types of exercise. Participant responses will be used to characterize overall activity behaviors and converted to METs, when possible, based on published values ${ }^{37}$ and the intensity of participation.

Symptom Monitoring Questionnaire. Participants are also asked 123 questions to report symptoms on a scale of mild-tosevere in each of the following systems: eyes/ears/nose/throat, gastrointestinal (including mouth), neurologic and muscular, psychologic/mental health, cardiopulmonary, skin, genitourinary, and constitutional.

\section{Statistical analytical plan}

The overall design of the proposed study, that is, a longitudinal cohort study, allows for both cross-sectional and longitudinal analyses. The authors will address their specific aims over time utilizing a priori statistical modeling to test their hypotheses in a stepwise manner.
To meet the first aim, the authors will primarily report descriptive statistics (frequency/\% and mean, median, range, standard deviation) of participant health indicators (e.g., frequency of medical conditions, laboratory values, and so on), dietary habits (e.g., macronutrient intake, \% eating organic, and so on), and self-care activities (e.g., physical activity, meditation, yoga, and so on). Subgroups will be created and compared by sex, year of education, and other demographic variables. Upon availability, participant baseline habit and health data will be discussed in the context of findings from other national and international cohort studies that included medical students and similarly aged adults.

For their second aim, multivariable linear regression models will be developed that assess for potential relationships between lifestyle factors and gut microbiome reports. Lifestyle variables will include measures of nutritional intake, physical activity, and mind-body practices, including meditation, yoga, acupuncture, and so on. The authors will first characterize all diet and nutritional related exposures using descriptive statistics. The VioScreen platform, used for FFQ collection, calculates respondents' Healthy Eating Index scores. The authors will also characterize the utilization of mind-body practices with descriptive statistics (e.g., number of minutes spent exercising or meditating per week, percentage of participants engaging in mindfulness, and so on). Finally, the authors will assess the relationship between potential coping mechanisms such as substance use and participant gut microbiome health. The authors will then test for associations with multivariable linear regression models, with Model 1 adjusting for age, sex, and race. Model 2 will add adjustments for medical history of major chronic disease or disability. Model 3 will then adjust for perceived stress score.

For their final aim, the authors will apply multivariable linear regression models to assess possible associations between perceived stress as an exposure variable (measured by the Perceived Stress Scale) and sleep (derived from the PROMIS-29 sleep subscore) and physical functioning (derived from the PROMIS-29 subscore) as outcomes. Model 1 will be a crude model, and Model 2 will adjust for age, sex, and race. Model 3A will add adjustment for medical history of major chronic disease or disability. Model 3B will then adjust for frequency and type of stress-management practices. Model 4 will add both medical history and stress management. A final model, Model 5, assessing physical functioning will also adjust for sleep quality.

Although the current protocol will focus on meeting the aims specified here, future protocol amendments will be proposed to address specific research questions as ancillary studies to the parent cohort. As the sample size of the cohort grows, additional analyses will become more feasible once the cohort structure is established. Although a primary objective of this cohort study is collect data on exposures to various forms of $\mathrm{CIH}$, and therefore all participants are likely to have some exposure to $\mathrm{CIH}$ generally, internal comparisons remain possible by comparing subgroups with and without exposure to individual practices, for example, those with meditative practices versus those without. These subgroup analyses will be age- and sex matched when possible, but more likely is to statistically adjust for key demographic differences and other potential confounders, for example, overall physical activity when considering 
mind-body relationships with stress, etc. These internal contrasts will become more powerful as the cohort sample grows over time.

\section{Discussion}

The results of this study will capture an unprecedented characterization of the health and wellness of students enrolled in colleges and universities with a $\mathrm{CIH}$ focus or interest. This protocol, designed to be pragmatic, has been relatively low cost and simple to implement at the flagship $\mathrm{CIH}$-focused institution. There is potential for a wealth of descriptive and prospective information regarding students, their CIH utilization, and potential health implications. The information garnered from INCLD Health will inform future $\mathrm{CIH}$ research and may foster new interventions for future studies.

The INCLD Health cohort structure also provides the opportunity to pilot and test the validation of measures that more accurately describe $\mathrm{CIH}$ practices and ideology against well-validated and accepted measures (e.g., MDI, PROMIS29, IPAQ, and so on). The authors have also developed novel questionnaires that will capture the unique lifestyle practices of this community and have included the appropriate aforementioned validated measures that will allow for comparison with standard guidelines, as well as validation in the future. In this way, the authors have developed a study that accepts the current state of the CIH community and anticipates the need to quantify and otherwise investigate how these lifestyle choices influence health.

Another strength of this study is the opportunity to include students in the entire process of conducting research as participants and research assistants. Engaging with the students in this way provides an opportunity for students to participate in evidence-based thinking on campus, as well as providing real-time experience in a process that may have been previously foreign (i.e., conducting longitudinal cohort studies). It is their aspiration to implement this protocol at a variety of institutions to foster more enthusiasm and understanding for scientific inquiry about self-care practices and $\mathrm{CIH}$ use in the student community.

A major limitation of the proposed study is the potential for participant survey burden, which may be a deterrent for students otherwise busy with coursework. At-home study specimen collection, while convenient, may be a limiting component due to the reliance on participants for returning the specimens to the study team; similarly, the need for specimens to be collected correctly and on certain days of the month for menstruating participants may be challenging.

Future directions will include the launch of INCLD Health internationally, as well as the launch of small ancillary investigations at the flagship site. Several sites across the globe have expressed interest and intend to pursue IRB approval at their institution in the coming months. Based on the flexible and low-personnel burden style of the INCLD Health study visits, the authors are confident that this protocol will be implemented with relative ease.

\section{Conclusion}

The INCLD Health study represents an opportunity to capture a wealth of health and lifestyle information about $\mathrm{CIH}$ utilization and self-care within a unique population of students in an innovative pragmatic manner after completing at least 1-6 years of CIH education. INCLD Health also presents the opportunity to test the validity of several unique measures of CIH-related lifestyle choices and habits and assess potential associations between these behaviors and well-established indicators of health status. As this protocol is implemented at institutions around the world, additional insights and unexpected prospects for self-care, wellness, and $\mathrm{CIH}$ research and education can be realized, including cross-cultural and cross-discipline comparisons.

\section{Authors' Contribution}

R.B., C.K.P., J.H., E.M., J.S., A.S., and J.A. contributed to this body of work based upon the initial conception and continued design of the INCLD Health Study. C.K.P., R.S.W., L.B., H.S., J.M., and M.T.T. assisted with data collection and analysis. C.K.P., R.S.W., and R.B. were responsible for drafting and revising the current article. All authors participated in the final approval of the version to be published.

\section{Author Disclosure Statement}

No competing financial interests exist.

\section{Funding Information}

Funding for the protocol thus far has been provided by the Helfgott Research Institute of the National University of Natural Medicine.

\section{Supplementary Material}

Supplementary Appendix SA1

\section{References}

1. Farhud DD. Impact of lifestyle on health. Iran J Public Health 2015;44:1442-1444.

2. Byrne DW, Rolando LA, Aliyu MH, et al. Modifiable healthy lifestyle behaviors: 10-Year health outcomes from a Health Promotion Program. Am J Prev Med 2016;51: 1027-1037.

3. Mehta N, Myrskylä M. The population health benefits of a healthy lifestyle: Life expectancy increased and onset of disability delayed. Health Aff (Millwood) 2017;36:14951502.

4. Sternfeld B, Colvin A, Stewart A, et al. The effect of a healthy lifestyle on future physical functioning in midlife women. Med Sci Sports Exerc 2017;49:274-282.

5. NHIS. National Health Interview Survey. Published October 9, 2020. Online document at: https://www.cdc.gov/ nchs/nhis/index.htm, accessed October 15, 2020.

6. NHANES. National Health and Nutrition Examination Survey Homepage. Published October 13, 2020. Online document at: https://www.cdc.gov/nchs/nhanes/index.htm, accessed October 15, 2020.

7. CDC. CDC Healthy Living. Centers for Disease Control and Prevention. Published January 29, 2020. Online document at: https://www.cdc.gov/healthyliving/index.html, accessed October 15, 2020.

8. History | Nurses' Health Study. Online document at: https://www.nurseshealthstudy.org/about-nhs/history, accessed October 15, 2020. 
9. Boston 677 Huntington Avenue, Ma $02115+1495-1000$ About the Study. Health professionals follow-up study. Published September 21, 2017. Online document at: https:// sites.sph.harvard.edu/hpfs/about-the-study, accessed October 15, 2020.

10. Health Professionals Follow-up Study-Full Text ViewClinicalTrials.gov. Online document at: https://clinicaltrials .gov/ct2/show/NCT00005182, accessed October 15, 2020.

11. Colditz GA, Manson JE, Hankinson SE. The Nurses' Health Study: 20-year contribution to the understanding of health among women. J Womens Health 1997;6:49-62.

12. Lee-Kwan SH. Disparities in state-specific adult fruit and vegetable consumption-United States, 2015. MMWR Morb Mortal Wkly Rep. 2017;66:3.

13. FastStats. Published August 10, 2020. Online document at: https://www.cdc.gov/nchs/fastats/exercise.htm, accessed October 15, 2020.

14. Eisenberg DM, Cohen MH, Hrbek A, et al. Credentialing complementary and alternative medical providers. Ann Intern Med 2002;137:965-973.

15. Hayes M, Muhota J, Nguyen L, et al. A framework for credentialing naturopathic physicians in academic health centers: Oregon Health and Science University. J Altern Complement Med N Y N 2014;20:217-218.

16. Caruana EJ, Roman M, Hernández-Sánchez J, Solli P. Longitudinal studies. J Thorac Dis 2015;7:E537E540.

17. von Elm E, Altman DG, Egger M, et al. Strengthening the reporting of observational studies in epidemiology (STROBE) statement: Guidelines for reporting observational studies. BMJ 2007;335:806-808.

18. Craig CL, Marshall AL, Sjöström M, et al. International physical activity questionnaire: 12-country reliability and validity. Med Sci Sports Exerc 2003;35:1381-1395.

19. Sanda B, Vistad I, Haakstad LAH, et al. Reliability and concurrent validity of the International Physical Activity Questionnaire short form among pregnant women. BMC Sports Sci Med Rehabil 2017;9:7.

20. Wani RT, Nabi SS. Reliability and validity of a culturally adaptive version of the International Physical Activity Questionnaire in Indian Subcontinent: A Cross-sectional Study. Int J Prev Med 2020;11:40.

21. Lou X, He Q. Validity and reliability of the International Physical Activity Questionnaire in Chinese Hemodialysis Patients: A Multicenter Study in China. Med Sci Monit Int Med J Exp Clin Res 2019;25:9402-9408.

22. Kurtze N, Rangul V, Hustvedt B-E. Reliability and validity of the international physical activity questionnaire in the Nord-Trøndelag health study (HUNT) population of men. BMC Med Res Methodol 2008;8:63.

23. Bech P, Timmerby N, Martiny K, et al. Psychometric evaluation of the Major Depression Inventory (MDI) as depression severity scale using the LEAD (Longitudinal Expert Assessment of All Data) as index of validity. BMC Psychiatry 2015;15:190.

24. Hovaldt HB, Nielsen T, Dammeyer J. Validity and reliability of the major depression inventory for persons with dual sensory loss. Innov Aging 2018;2:igy010.
25. Fountoulakis K, Iacovides A, Kleanthous S, et al. Reliability, validity and psychometric properties of the Greek translation of the Major Depression Inventory. BMC Psychiatry $2003 ; 3: 2$.

26. Cella D, Riley W, Stone A, et al. The Patient-Reported Outcomes Measurement Information System (PROMIS) developed and tested its first wave of adult self-reported health outcome item banks: 2005-2008. J Clin Epidemiol 2010;63:1179-1194.

27. Kroenke K, Yu Z, Wu J, et al. Operating characteristics of PROMIS Four-Item Depression and Anxiety Scales in primary care patients with chronic pain. Pain Med Off J Am Acad Pain Med 2014;15:1892-1901.

28. Rose AJ, Bayliss E, Huang W, et al. Evaluating the PROMIS-29 v2.0 for use among older adults with multiple chronic conditions. Qual Life Res Int J Qual Life Asp Treat Care Rehabil 2018;27:2935-2944.

29. Weinfurt KP, Lin L, Bruner DW, et al. Development and initial validation of the PROMIS ${ }^{\circledR}$ Sexual Function and Satisfaction Measures Version 2.0. J Sex Med 2015;12: 1961-1974.

30. Lee E-H. Review of the psychometric evidence of the perceived stress scale. Asian Nurs Res 2012;6:121-127.

31. McDonald D, Hyde E, Debelius JW, et al. American Gut: An open platform for citizen science microbiome research. mSystems 2018;3:e0031-18.

32. VioScreen. VIOCARE ${ }^{\circledR}$. Online document at: https://www .viocare.com/vioscreen.html, accessed October 15, 2020.

33. Kristal AR, Kolar AS, Fisher JL, et al. Evaluation of WebBased, Self-Administered, Graphical Food Frequency Questionnaire. J Acad Nutr Diet 2014;114:613-621.

34. pwi-a-english.pdf. Online document at: www.acqol.com .au/uploads/pwi-a/pwi-a-english.pdf, accessed October 15, 2020.

35. Mbizo J, Okafor A, Sutton MA, et al. Complementary and alternative medicine use among persons with multiple chronic conditions: Results from the 2012 National Health Interview Survey. BMC Complement Altern Med 2018;18:281.

36. Third National Health and Nutrition Examination Survey (NHANES III), Centers for Disease Control and Prevention (CDC). National Center for Health Statistics (NCHS). National Health and Nutrition Examination Survey Data. Hyattsville, MD: U.S. Department of Health and Human Services, Centers for Disease Control and Prevention, 1988-94.

37. Ainsworth BE, Haskell WL, Herrmann SD, et al. 2011 Compendium of Physical Activities: A second update of codes and MET values. Med Sci Sports Exerc 2011;43: 1575-1581.

Address correspondence to: Ryan Bradley, ND, MPH Helfgott Research Institute National University of Natural Medicine 2220 SW 1st Avenue Portland, OR 97201 USA

E-mail:rbradley@nunm.edu 